\title{
A snapshot beyond the Local Universe with Herschel/SPIRE
}

\author{
Sandor Pinter ${ }^{1}$, Gabor Marton ${ }^{2}$, L. Viktor Toth ${ }^{1}$, \\ Chris Pearson ${ }^{3}$, Zsolt Bagoly ${ }^{1}$, Lajos G. Balazs ${ }^{1,2}$, \\ Istvan Horvath ${ }^{4}$, Istvan I. Racz ${ }^{1}$ \\ ${ }^{1}$ Eotvos Lorand University, Hungary, \\ email: s.pinter@astro.elte.hu \\ ${ }^{2}$ Konkoly Observatory of the Hungarian Academy of Sciences \\ ${ }^{3}$ Science and Technology Facilities Council Rutherford Appleton Laboratory. \\ ${ }^{4}$ National University of Public Service, Hungary
}

\begin{abstract}
The European Large-Area ISO Survey (ELAIS) N1 field is one of the extragalactic windows where the lack of the Galactic ISM allows us to analyze the unbiased FIR properties of extragalactic objects. The field was investigated recently based on Herschel observations by the HerMES key project. We present a survey of the field covering a larger area than HerMES $\left(12.54 \mathrm{deg}^{2}\right.$ vs $\left.3.47 \mathrm{deg}^{2}\right)$. We provide accurate 250,350, and 500 micrometer flux densities for about 8000 point sources using the latest Herschel analysis and calibration procedures. Based on SDSS spectroscopic and SWIRE photometric data our sample has approximately 4000 and 4500 galaxies with $0.2<z<0.5$ and $z>0.5$ redshifts, respectively. The new flux densities are crucial limiting the star-forming activity of galaxies outside the Local Universe, as it is demonstrated in the star-forming galaxy 2MASS J16072472+5412119.
\end{abstract}

Keywords. galaxies: starburst, infrared: galaxies, galaxies: individual (ELAIS N1-015)

Our objective was to put constraints on the star forming properties of galaxies using Herschel FIR fluxes. We used the SPIRE 250, 350, and $500 \mu \mathrm{m}$ Level 2.5 maps of newer Herschel observations (IDs: 1342228354 \& 1342228450) processed using the HIPE v13 (Ott 2010). The flux of each source was measured with the SUSSEXtractor task (Pearson et al. 2014). The new SPIRE fluxes are crucial to determine the cold dust properties of high redshift galaxies. The redshifts was acquired from the SWIRE catalog (Rowan-Robinson et al. 2013). We show the SED of the infrared galaxy ELAIS N1-015 as an example in Fig.1. which is a star forming galaxy with SFR $=36 \pm 13 \mathrm{M}_{\odot} \mathrm{yr}^{-1}$ (Sajina et al. 2006). We note that neither the ISOPHOT $170 \mu m$ nor the SCUBA $850 \mu m$ data point fit well, but the cold dust emission part of the fitted SED is now confirmed by our new photometry.

\section{Acknowledgements}

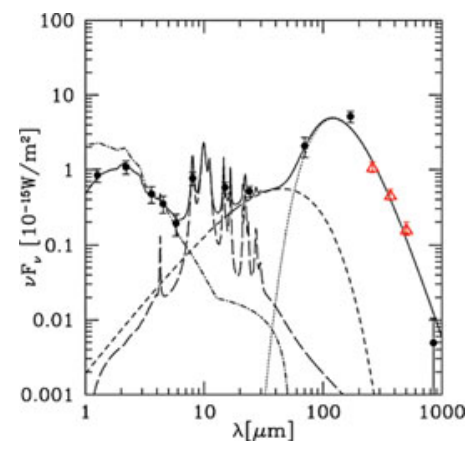

Figure 1. The SED of the 2MASS J16072472+5412119. This includes a greybody (dotted line), a warm power law (short-dashed line), PAH emission (longdashed line), and unextincted stellar emission (dotdashed line) with $e^{-\tau \nu}$ extinction applied. The thick solid line is the total. The triangles are the SPIRE fluxes.

Herschel is an ESA space observatory with science instruments provided by Europeanled Principal Investigator consortia and with important participation from NASA. Our research was funded by Hungarian Research Fund (OTKA) grants nr. K104607, K101393, 
and NN111016. The work of G. Marton has been supported by PECS contract no. 4000109997/13/NL/KML of the Hungarian Space Office and the European Space Agency.

\section{References}

Oliver, S. et al. 2012 MNRAS, 424, 1614

Ott, S. 2010 ASP Conference, 434, 139

Pearson, C. et al. 2014 Exp. Astr., 37, 175

Rowan-Robinson, M. et al. 2013 MNRAS, 428, 1958

Sajina, A. et al. 2006 MNRAS, 369, 939 